EPJ Web of Conferences 109,02002 (2016)

DOI: $10.1051 /$ epjconf/201610902002

C) Owned by the authors, published by EDP Sciences, 2016

\title{
A Chemical Evolution Model for the Fornax Dwarf Spheroidal Galaxy
}

\author{
Zhen Yuan ${ }^{1,2, a}$, Yong-Zhong Qian ${ }^{1,3}$, and Yi Peng Jing ${ }^{2}$ \\ ${ }^{1}$ School of Physics and Astronomy, University of Minnesota, Minneapolis, MN 55455, USA \\ ${ }^{2}$ Center for Astronomy and Astrophysics, Department of Physics and Astronomy, Shanghai Jiao Tong Uni- \\ versity, Shanghai 200240, China \\ ${ }^{3}$ Center for Nuclear Astrophysics, INPAC, Department of Physics and Astronomy, Shanghai Jiao Tong Uni- \\ versity, Shanghai 200240, China
}

\begin{abstract}
Fornax is the brightest Milky Way (MW) dwarf spheroidal galaxy and its star formation history (SFH) has been derived from observations. We estimate the time evolution of its gas mass and net inflow and outflow rates from the SFH using a simple star formation law that relates the star formation rate to the gas mass. We present a chemical evolution model on a 2D mass grid with supernovae ( $\mathrm{SNe}$ ) as sources of metal enrichment. We find that a key parameter controlling the enrichment is the mass $M_{x}$ of the gas to mix with the ejecta from each SN. The choice of $M_{x}$ depends on the evolution of SN remnants and on the global gas dynamics. It differs between the two types of $\mathrm{SNe}$ involved and between the periods before and after Fornax became an MW satellite at time $t=t_{\text {sat }}$. Our results indicate that due to the global gas outflow at $t>t_{\text {sat }}$, part of the ejecta from each SN may directly escape from Fornax. Sample results from our model are presented and compared with data.
\end{abstract}

\section{Introduction}

With the advent of large telescopes and supercomputers, studies of galaxy formation and evolution have entered a new era. In the meantime, there has also been major progress in our understanding of stellar explosion and nucleosysnthesis. Elemental abundances in stars form an important link between galactic and stellar processes. Because the surface abundances of long-lived low-mass stars record the composition of their birth environments, they serve as fossils of galactic chemical evolution. By studying abundances in stars of different ages in an individual galaxy, we can obtain valuable information on its formation and evolution. Here we focus on nearby dwarf spheroidal galaxies (dSphs), which are considered to be surviving building blocks of larger galaxies in the framework of hierarchical structure formation. Understanding how $\mathrm{dSphs}$ evolve is crucial to unraveling the formation and evolution of larger galaxies such as the Milky Way (MW). Unlike larger galaxies that have undergone frequent mergers, dSphs have a smoother evolution, which makes them a good laboratory to study various physical processes on galactic scales.

As an example of the general approach, we first estimate the time evolution of the gas mass and net inflow and outflow rates from the star formation history (SFH) for Fornax, the brightest MW dSph.

ae-mail: yuan@physics.umn.edu 
We then build a model for its chemical evolution and compare the results with stellar abundance data. Observations strongly indicate that chemical enrichment in Fornax remains inhomogeneous until the very end of its SFH despite its relatively small size. Using nucleosynthetic yields from both core-collapse (CCSNe) and Type Ia supernovae (SNe Ia), we numerically simulate the stochastic and inhomogeneous mixing of newly-synthesized elements in a 2D disk system. By comparing the resulting abundance distributions with data on Fornax, we find that the mixing depends on large-scale gas flows and the environments surrounding CCSNe and SNe Ia.

\section{Global Gas Dynamics of Fornax}

We assume a star formation law of the Schmidt-Kennicutt form:

$$
\frac{\Sigma_{\mathrm{SFR}}}{M_{\odot} \mathrm{yr}^{-1} \mathrm{kpc}^{-2}}=C\left(\frac{\Sigma_{g}}{10 M_{\odot} \mathrm{pc}^{-2}}\right)^{\alpha}
$$

where $\Sigma_{\mathrm{SFR}}$ is the star formation rate (SFR) per unit area, $\Sigma_{g}$ is the surface density of gas, and $C$ and $\alpha$ are constants. We consider that star formation in Fornax occurred in a uniform disk, the area $A_{\text {disc }}(t)$ of which grew until Fornax became an MW satellite at time $t=t_{\text {sat }}$ and then stayed fixed until the end of star formation. Under the above assumptions, the global SFR $\psi(t)$ can be written as

$$
\psi(t)=\lambda_{*}(t)\left[\frac{M_{g}(t)}{M_{\odot}}\right]^{\alpha}
$$

where $M_{g}$ is the total mass of gas in the disk, and

$$
\lambda_{*}(t)=C\left[\frac{10 \mathrm{kpc}^{2}}{A_{\mathrm{disc}}(t)}\right]^{\alpha-1} \times 10^{1-8 \alpha} M_{\odot} \mathrm{yr}^{-1} .
$$

As $A_{\text {disc }}(t)=A_{\text {disc }}\left(t_{\text {sat }}\right)$ for $t \geq t_{\text {sat }}$, the above equation can be rewritten as

$$
\lambda_{*}(t)= \begin{cases}\lambda_{*}\left(t_{\mathrm{sat}}\right)\left[A_{\mathrm{disc}}\left(t_{\mathrm{sat}}\right) / A_{\mathrm{disc}}(t)\right]^{\alpha-1}, & t<t_{\mathrm{sat}}, \\ C\left[10 \mathrm{kpc}^{2} / A_{\mathrm{disc}}\left(t_{\mathrm{sat}}\right)\right]^{\alpha-1} \times 10^{1-8 \alpha} M_{\odot} \mathrm{yr}^{-1}, & t \geq t_{\mathrm{sat}} .\end{cases}
$$

We estimate $A_{\text {disc }}\left(t_{\mathrm{sat}}\right) \sim 10 \mathrm{kpc}^{2}$ from the radial distribution of stars in the present-day Fornax [1]. In accord with values of $C$ and $\alpha$ derived from observations of star formation in nearby faint dwarf irregular galaxies [2], we take $\alpha=1.5$ and $\lambda_{*}\left(t_{\text {sat }}\right)=8 \times 10^{-15} M_{\odot} \mathrm{yr}^{-1}$ for the presentation below.

We use Eq. (2) to estimate $M_{g}$ from Fornax's SFH as

$$
M_{g}(t)=\left[\frac{\psi(t)}{\lambda_{*}(t)}\right]^{1 / \alpha} M_{\odot}
$$

We further estimate the net gas flow rate as

$$
\Delta F(t) \equiv \frac{d M_{g}}{d t}+\psi(t)
$$

We expect a net inflow, i.e., $\Delta F>0$ for $t<t_{\text {sat }}$ as gas was accreted along with dark matter during the growth of the halo hosting Fornax. The halo stopped growing when it became an MW satellite. Subsequently, its tidal interaction with the MW and the ram pressure associated with its orbital motion 
worked together to remove gas from Fornax. So we expect a net outflow, i.e., $\Delta F<0$ for most of the time $t>t_{\text {sat }}$. To estimate $t_{\text {sat }}$, we first assume a fixed $\lambda_{*}(t)=\lambda_{*}\left(t_{\text {sat }}\right)$ for all $t$. Using the data on $\psi(t)$ [1], we find that the growth of $M_{g}$ slowed down greatly (Fig. 1a) and $\Delta F$ started to decrease towards zero for the first time (Fig. 1b) at $t \approx 4.8 \mathrm{Gyr}$. If we take this time as the estimate for $t_{\text {sat }}$, then $d \psi / d t>0$ and $d \lambda_{*} / d t<0$ ensure that $\Delta F>0$ for $t<t_{\text {sat }}$ even when we allow $\lambda_{*}(t)$ to change as in Eq. (4). We adopt $t_{\text {sat }} \approx 4.8$ Gyr.

Using the median halo growth history given by Ref. [3], we estimate that the Fornax halo grew to a total mass of $M_{h} \approx 1.8 \times 10^{9} M_{\odot}$ at $t_{\mathrm{sat}} \approx 4.8 \mathrm{Gyr}$ in order to obtain the mass enclosed within the half-light radius inferred from observations [4]. Taking $A_{\mathrm{disc}}(t) / A_{\mathrm{disc}}\left(t_{\mathrm{sat}}\right)=\left[r_{\mathrm{vir}}(t) / r_{\mathrm{vir}}\left(t_{\mathrm{sat}}\right)\right]^{2}$ for $t<t_{\text {sat }}$, where $r_{\text {vir }}$ is the virial radius of the Fornax halo, we show the corresponding evolution of $M_{g}$ and $\Delta F$ in Fig. 1 (see Refs. [5, 6] for more details). The full evolution of $M_{g}$ and $\Delta F$ will be used to build a chemical evolution model for Fornax below.
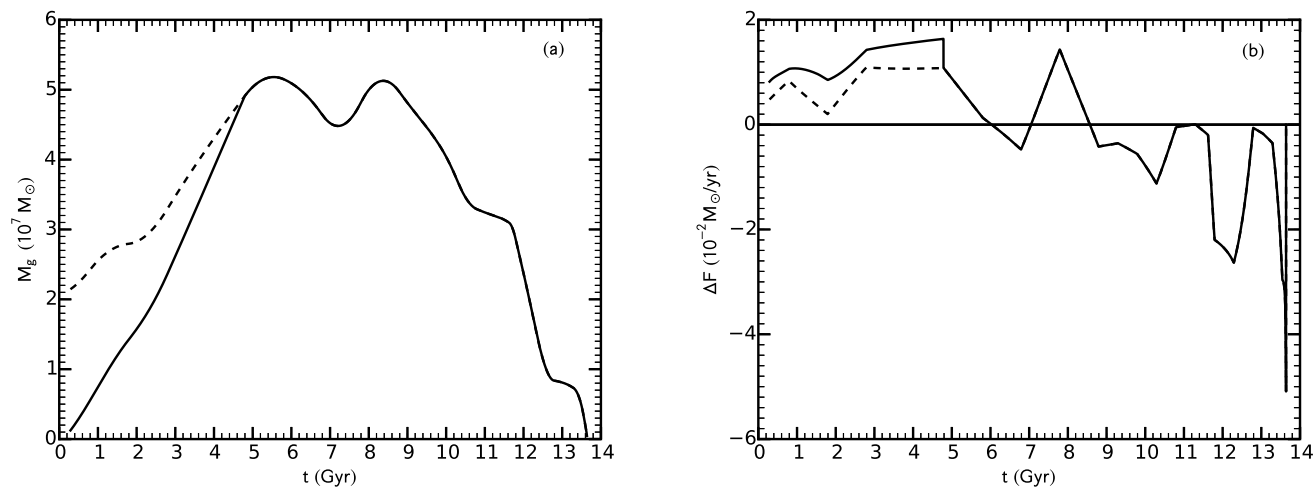

Figure 1. Evolution of (a) the gas mass $M_{g}$ and (b) the net gas flow rate $\Delta F$ as functions of time $t$ for Fornax. We estimate that Fornax became an MW satellite at $t_{\text {sat }} \approx 4.8 \mathrm{Gyr}$. The solid curves correspond to a star-forming disk that grew until $t_{\mathrm{sat}}$ and then stayed fixed. The dashed curves assume the same disk size before and after $t_{\mathrm{sat}}$.

\section{Simulating Chemical Evolution of Fornax}

Fornax is $\sim 10^{3}$ times smaller than the MW and has a prolonged SFH that lasted almost the age of the universe. Fornax is also chemically much more inhomogeneous [1] than the MW disk although both systems have evolved for approximately the same amount of time. This apparently counterintuitive result can be understood from the efficiency for mixing of the interstellar medium by $\mathrm{SNe}$ in a gaseous system of mass $M_{g}$. Assuming that a total mass $M_{x}$ of the interstellar medium is mixed by an SN, we estimate that the rate for the fraction of gas mixed is $f_{\text {mix }} \propto \psi M_{x} / M_{g} \propto M_{g}^{\alpha-1}$, which increases with $M_{g}$ because $\alpha>1$. This suggests that the larger a system is, the more quickly it would become chemically homogeneous. In addition, the rate for enrichment of an element $\mathrm{E}$ can be estimated as $f_{\mathrm{E}} \propto \psi\left\langle Y_{\mathrm{E}}\right\rangle / M_{g} \propto M_{g}^{\alpha-1}$, where $\left\langle Y_{\mathrm{E}}\right\rangle$ is the average SN yield of E. Consequently, a larger system such as the MW is more chemically enriched than Fornax and other dSphs [7] even if the duration of enrichment is the same.

To simulate the chemical evolution of Fornax, we use a 2D mass grid with $n=10^{3} \times 10^{3}$ boxes to approximate its gaseous disk for star formation. The number of boxes filled with gas changes with 
time according to the gas mass $M_{g}$ shown as the solid curve in Fig. 1a. Each gas box contains a fixed mass $m_{0}=M_{g \text {, } \max } / n$, where $M_{g \text {,max }}$ is the maximum gas mass. We take time steps of $0.2 \mathrm{Gyr}$ each. For the $i$ th time step $t_{i-1} \leq t<t_{i}(i \geq 1)$, we first treat the gas inflow or outflow by adding or dropping gas boxes, respectively, so that the number of gas boxes is $n_{i}=M_{g}\left(t_{i}\right) / m_{0}$. We assume that the inflow is metal free during the pre-satellite phase $\left(t<t_{\text {sat }}\right)$ and for $t_{\text {sat }}<t \lesssim 6 \mathrm{Gyr}$ during the satellite phase. The inflow for $7 \lesssim t \lesssim 8.6 \mathrm{Gyr}$ consists of reaccreted gas and its metallicity is calculated from our model. We calculate the numbers $n_{\mathrm{CC}, i}$ and $n_{\mathrm{Ia}, i}$ of CCSNe and SNe Ia, respectively, for the $i$ th time step based on the SFH. The fraction of stars resulting in CCSNe is estimated from the initial mass function given by Ref. [8]. The birth rate for progenitor systems of SNe Ia is specified as a fraction of the CCSN rate. The delay between the birth of an SN Ia progenitor system and its explosion is assumed to follow the distribution given by Ref. [9]. We then randomly pick $n_{\mathrm{CC}, i}$ and $n_{\mathrm{I}, i}$ from a total of $n_{i}$ boxes as sites of CCSNe and SNe Ia, respectively. Both types of SNe inject newly-synthesized elements into the surrounding gas boxes. The mixing and chemical enrichment of the gas surrounding each SN is described below. To finish the $i$ th time step, we randomly sample $n_{s, i}$ boxes, where $n_{s, i}$ is the number of stars that were formed during this time step and survive to the present based on the SFH and stellar lifetimes. The above procedure is repeated for the entire SFH.

The elemental yields of CCSNe and SNe Ia are taken from Refs. [10] and [11], respectively. A specific SN enriches and mixes the gas in a total of $n_{x}=M_{x} / m_{0}$ surrounding boxes. The resulting mass fraction of element $\mathrm{E}$ in these boxes is

$$
X_{\mathrm{E}, j}^{\prime}=\frac{m_{0} \sum_{k=1}^{n_{x}} X_{\mathrm{E}, k}+Y_{\mathrm{E}}}{M_{x}}, j=1,2, \cdots, n_{x},
$$

where $X_{\mathrm{E}, k}$ is the mass fraction of $\mathrm{E}$ in the $k$ th box before the $\mathrm{SN}$ occurs and $Y_{\mathrm{E}}$ is the yield of the $\mathrm{SN}$. In view of the dominance of the outflow during the satellite phase, $Y_{\mathrm{E}}$ is reduced for this phase by a factor of 2 or 4 from the theoretical yield for a CCSN or SN Ia, respectively, to account for approximately the direct loss of metals from each SN. The total mass $M_{x}$ of the gas to mix with each $\mathrm{SN}$ is estimated from the results in Ref. [12]. As CCSNe are associated with active star-forming regions of high densities but $\mathrm{SNe}$ Ia tend to occur in regions of low densities, we set the ratio of the mixing masses for each SN Ia and CCSN to be $M_{x, \mathrm{Ia}} / M_{x, \mathrm{CC}}=5$ throughout the SFH. The mixing mass is also affected by the global gas flow. During the phase dominated by the inflow, cold dense gas falls into the system while hot "bubbles" are blown out by SNe. This would drive significant turbulence on the galactic scale [13] and result in widespread mixing of gas. In contrast, SN bubbles cannot survive for long during the phase dominated by the outflow, which suggests inefficient mixing for this phase. Based on the above discussion, we set $M_{x, \mathrm{Ia}}=10^{5} M_{\odot}, M_{x, \mathrm{CC}}=2 \times 10^{4} M_{\odot}$ during the pre-satellite phase, and $M_{x, \mathrm{Ia}}=5 \times 10^{3} M_{\odot}, M_{x, \mathrm{CC}}=10^{3} M_{\odot}$ during the satellite phase.

\section{Results}

A snapshot of the chemical composition in each gas box is taken at the end of each time step. These snapshots describe the inhomogeneous chemical evolution of Fornax. In particular, snapshots of gas boxes sampled by simulated stars that were formed during each time step and survive until today can be compared with observations. Here we only present sample results on $[\alpha / \mathrm{Fe}]$ vs. $[\mathrm{Fe} / \mathrm{H}]$, where $\alpha$ stands for $\mathrm{Mg}, \mathrm{Si}, \mathrm{Ca}$, and $\mathrm{Ti}$ (see Ref. [6] for more details).

We compile the simulated stars in the increasing order of their $[\mathrm{Fe} / \mathrm{H}]$ values. At a specific $[\mathrm{Fe} / \mathrm{H}]$ value, we calculate the algebraic averages of $[\alpha / \mathrm{Fe}]$ and show these as the solid curves in Fig. 2 . We also determine the ranges of $[\alpha / \mathrm{Fe}]$ covered by $68 \%, 95 \%$, and $99.7 \%$ of the stars, respectively. These ranges correspond to the $1 \sigma, 2 \sigma$ and $3 \sigma$ confidence regions, and are indicated (cumulatively) by the 
central white, the light grey, and the dark grey regions, respectively, in Fig. 2 for $\mathrm{Mg}$, Si, and Ca. The stellar data [1, 14-17] are also shown for comparison. As most of the data on $\mathrm{Mg}, \mathrm{Si}$, and $\mathrm{Ca}$ lie within the $95 \%$ confidence regions, the scatter of the data can be approximately accounted for by our model. In addition, the general trends of these data can be described by the solid curves. We note that the agreement between our results and the data is the best for $\mathrm{Mg}$, which also happens to be the best measured $\alpha$ element. There are some data on $\mathrm{Si}$ and $\mathrm{Ca}$ that lie in the dark grey regions. How representative these data are should be determined by high-quality observations of more stars.

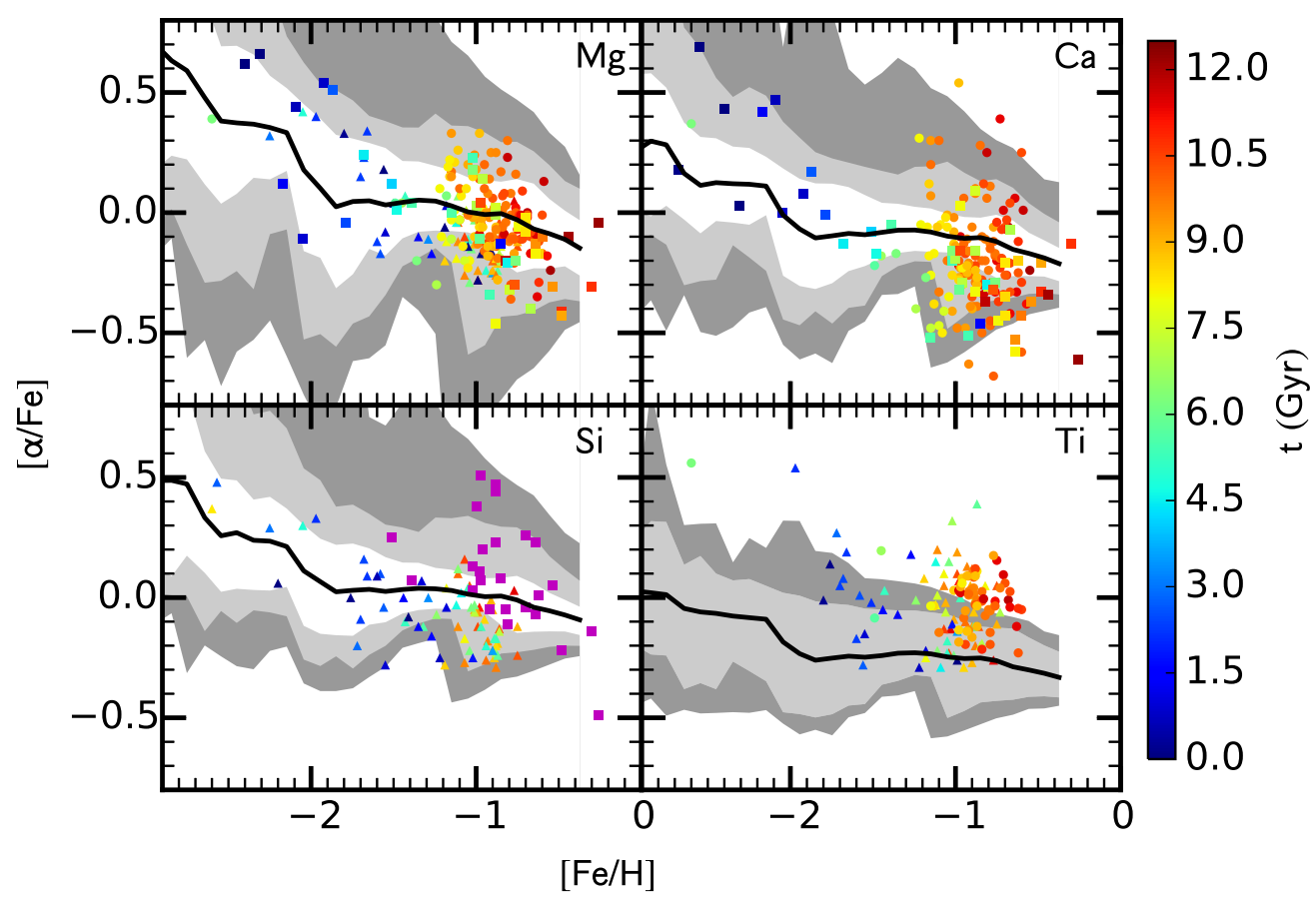

Figure 2. Comparison of simulation results with stellar data on $[\alpha / \mathrm{Fe}]$ vs. $[\mathrm{Fe} / \mathrm{H}]$ for Fornax. The solid curves represent the average $[\alpha / \mathrm{Fe}]$ values as functions of $[\mathrm{Fe} / \mathrm{H}]$ from simulations. The central white, the light grey, and the dark grey regions indicate (cumulatively) the $1 \sigma, 2 \sigma$, and $3 \sigma$ confidence regions, respectively, for $\mathrm{Mg}, \mathrm{Si}$, and Ca. Only the $2 \sigma$ and $3 \sigma$ confidence regions are shown for Ti. Data are taken from Refs. $[1,14,15]$ (circles), [16] (squares), and [17] (triangles). Colors indicate the formation time of stars except for the purple squares, for which no such information is available. Note the general agreement for $\mathrm{Mg}, \mathrm{Si}$, and $\mathrm{Ca}$ as well as the offset for Ti between simulation results and data. The offset for Ti may be due to systematic underestimates of Ti yields by CCSN models for some unknown reasons or to systematic uncertainties in measurements of Ti abundances.

The solid curve for Ti lies below most of the data. In addition, because Ti and Fe are coproduced by explosive nucleosynthesis with similar yield ratios for all CCSNe, the $1 \sigma$ confidence region for $[\mathrm{Ti} / \mathrm{Fe}]$ vs. $[\mathrm{Fe} / \mathrm{H}]$ is rather difficult to determine. Therefore, we only show the $2 \sigma$ and $3 \sigma$ confidence regions in Fig. 2. It can be seen that both the solid curve and the confidence regions are offset from the data on Ti by $\sim 0.3$ dex. This may indicate that the Ti yields are systematically underestimated by 
CCSN models for some unknown reasons. It is also possible that the yield ratios of Ti to Fe have much larger variations among CCSNe than estimated by current models. We have examined the effects on $[\mathrm{Ti} / \mathrm{Fe}]$ vs. $[\mathrm{Fe} / \mathrm{H}]$ by including SN Ia contributions to both Ti and Fe based on several models [11] and found that the problem persists or even worsens. Nevertheless, it is worthwhile to explore SN Ia yields of $\mathrm{Ti}$ and $\mathrm{Fe}$ with more models. Finally, observational uncertainties should be examined because there also appears to be a systematic offset between Ti abundances determined with Ti I and Ti II in different ionization states.

As can be seen clearly from Fig. 2 , the $2 \sigma$ confidence regions shrink significantly at $[\mathrm{Fe} / \mathrm{H}] \sim-1.4$, especially for $\mathrm{Mg}, \mathrm{Si}$, and $\mathrm{Ca}$. This results from the relatively efficient mixing of gas during the presatellite phase of Fornax and is consistent with the data. However, large scatter in the data appears again at higher $[\mathrm{Fe} / \mathrm{H}]$ values. We take this to indicate that mixing of gas was very inefficient during the satellite phase of Fornax, possibly due to the disruption of individual growing SN bubbles by the global gas outflow.

This work was supported in part by the US DOE (DE-FG02-87ER40328), the NSFC (11320101002), 973-Project 2015CB857000, Shanghai Key Laboratory Grant No. 11DZ2260700, and the CAS/SAFEA International Partnership Program for Creative Research Teams (KJCX2-YW$\mathrm{T} 23)$.

\section{References}

[1] T.J.L. de Boer, E. Tolstoy, V. Hill, A. Saha, E.W. Olszewski, M. Mateo, E. Starkenburg, G. Battaglia, M.G. Walker, A\&A544, A73 (2012)

[2] S. Roychowdhury, M.L. Huang, G. Kauffmann, J. Wang, J.N. Chengalur, MNRAS449, 3700 (2015)

[3] D.H. Zhao, Y.P. Jing, H.J. Mo, G. Börner, ApJ707, 354 (2009)

[4] J. Wolf, G.D. Martinez, J.S. Bullock, M. Kaplinghat, M. Geha, R.R. Muñoz, J.D. Simon, F.F. Avedo, MNRAS406, 1220 (2010)

[5] Z. Yuan, Y.Z. Qian, Y.P. Jing, ArXiv e-prints (2015), 1503.00780

[6] Z. Yuan, Ph.D. thesis, University of Minnesota (2015)

[7] E. Tolstoy, V. Hill, M. Tosi, ARA\&A47, 371 (2009)

[8] P. Kroupa, MNRAS322, 231 (2001)

[9] D. Maoz, F. Mannucci, T.D. Brandt, MNRAS426, 3282 (2012)

[10] A. Heger, S.E. Woosley, ApJ724, 341 (2010)

[11] K. Maeda, F.K. Röpke, M. Fink, W. Hillebrandt, C. Travaglio, F.K. Thielemann, ApJ712, 624 (2010)

[12] K. Thornton, M. Gaudlitz, H.T. Janka, M. Steinmetz, ApJ500, 95 (1998)

[13] A.C. Petit, M.R. Krumholz, N.J. Goldbaum, J.C. Forbes, MNRAS449, 2588 (2015)

[14] B. Letarte, V. Hill, E. Tolstoy, P. Jablonka, M. Shetrone, K.A. Venn, M. Spite, M.J. Irwin, G. Battaglia, A. Helmi et al., A\&A523, A17 (2010), 1007.1007

[15] E.N. Kirby, G.A. Lanfranchi, J.D. Simon, J.G. Cohen, P. Guhathakurta, ApJ727, 78 (2011)

[16] B. Lemasle, T.J.L. de Boer, V. Hill, E. Tolstoy, M.J. Irwin, P. Jablonka, K. Venn, G. Battaglia, E. Starkenburg, M. Shetrone et al., A\&A572, A88 (2014)

[17] B. Hendricks, A. Koch, M. Walker, C.I. Johnson, J. Peñarrubia, G. Gilmore, A\&A572, A82 (2014) 